Research Paper

\title{
Active Chronic Hepatitis B increases the risk of Colorectal Liver Metastasis - A retrospective cross-sectional study
}

\author{
Yue Yang ${ }^{1,2^{*}}$, Lijie Song $3^{*}$, Jingyu Cao ${ }^{4}$, Jing Liu ${ }^{5}$, Dongxu Wang ${ }^{6}$, Linda L. Wong7, Lei Zhao ${ }^{1,2 \bowtie}$ \\ 1. Shandong First Medical University and Shandong Academy of Medical Sciences, 6699 Qingdao road, Huaiyin District, Jinan, China. \\ 2. Department of Hepatobiliary Surgery, Shandong Cancer Hospital and Institute, Shandong First Medical University and Shandong Academy of Medical Sciences, 440 \\ Jiyan road, Huaiyin District, Jinan, China. \\ 3. Department of Oncology, The First Affiliated Hospital of Zhengzhou University, 2 Jianshe East Road, Erqi District, Zhengzhou, China. \\ 4. Department of Hepatobiliary Surgery, The Affiliated Hospital of Qingdao University, 1677 Wutaishan Road, Huangdao District, Qingdao, China. \\ 5. Department of Biostatistics, School of Public Health, Shandong University, 44 Wenhua West Road, Lixia District, Jinan, China. \\ 6. Department of Liver Surgery, Peking Union Medical College Hospital, Chinese Academy of Medical Sciences and Peking Union Medical College, No.1 Shuaifuyuan \\ Wangfujing Dongcheng District, Beijing, China. \\ 7. Department of Surgery, University of Hawaii School of Medicine, 3430 Keahi Place, Honolulu, HI96822, USA. \\ *These authors contributed equally to this work. \\ $\triangle$ Corresponding author: Lei Zhao, Department of Hepatobiliary Surgery, Shandong cancer Hospital and Institute, 440 Jiyan Road, Jinan, 250117, China; Phone: \\ +86-531-67626368; E-mail: drzhaolei@hotmail.com;drzhaolei@sdu.edu.cn. \\ (C) The author(s). This is an open access article distributed under the terms of the Creative Commons Attribution License (https://creativecommons.org/licenses/by/4.0/). \\ See http://ivyspring.com/terms for full terms and conditions.
}

Received: 2020.07.28; Accepted: 2020.12.04; Published: 2021.01.01

\begin{abstract}
Background: A considerable part of colorectal cancer (CRC) patients also have chronic hepatitis $B$ (CHB), esp. in Asia. The effect of concomitant active CHB on the hazard of colorectal liver metastasis (CRLM) remains unclear. To evaluate the effect of concomitant active CHB on the risk of CRLM.

Methods: The medical record of all newly diagnosed CRC patients who were hospitalized to the three hospitals between January 2010 to January 2016 were reviewed, the prevalence of synchronous CRLM (synCRLM) were retrospectively studied. Totally 7187 cases of newly diagnosed CRC, including 368 cases with concomitant $\mathrm{CHB}$ were recruited. The prevalence of synCRLM in $\mathrm{HBs} A g^{+} / \mathrm{HBeAg}^{+}$patients was compared to that in $\mathrm{HBsAg}^{+} / \mathrm{HBeAg}$ - patients. Significant risk factors for synCRLM were analyzed by logistic regression analysis.

Results: The overall prevalence of synCRLM was $8.72 \%(627 / 7187)$ and was significantly higher in $\mathrm{HBsAg}+$ patients (43/368) than HBsAg- patients (576/6742) (11.68\% vs. 8.54\%, $\mathrm{P}=0.037$; $\mathrm{X}^{2}$ test). In 368 $\mathrm{HBsAg}^{+}$patients, 365 patients also had $\mathrm{HBeAg}$ information. synCRLM was also more prevalent inHBsAg ${ }^{+} / \mathrm{HBeAg}^{+}$patients (13/69) compared to $\mathrm{HBsAg}^{+} / \mathrm{HBeAg}^{-}$patients $(30 / 296)(18.84 \%$ vs. $10.14 \%$, $P=0.043 ; X^{2}$ test). In univariate and multivariate logistic regression analysis, $\mathrm{HBeAg}$ positivity was the second strongest predictor of synCRLM (multivariate: $O R, 2.622, P=0.020$ ) after CEA. (univariate: $O R$, 2.920, $P=0.001)$.

Conclusions: $\mathrm{HBeAg}$ positivity is a clinical risk factor for CRLM that can be readily identified and addressed. Whether anti-CHB treatment can decrease the risk of CRLM worth carefully-designed prospective trials to define.
\end{abstract}

Key words: chronic hepatitis B (CHB); colorectal liver metastasis (CRLM); hepatitis B e antigen (HBeAg); risk

\section{Introduction}

Worldwide, colorectal cancer (CRC) is the fourth primary reason of cancer mortality and is increasing in medium to highly-developed countries especially in Asia and Europe. China has the highest number of new cases, accounting for about $28 \%$ of global CRC cases $[1,2]$.

Asia also has a heavy burden of viral hepatitis
B. It is estimated that the global prevalence of hepatitis B surface antigen (HBsAg) was 3.9\% in 2016 [3]. Among 9 Asian countries which each had more than 10,000 new CRC cases in 2018, the prevalence of HBsAg in China, Indonesia, Philippines and Vietnam were much higher than the average global level, while the HBsAg prevalence in India, Republic 
of Korea, Turkey, Thailand varied between 2.4 and $3.5 \%$ [4]. Although hepatitis B virus (HBV) prevention policy in China has significantly decreased the prevalence of $\mathrm{HBsAg}$ in the younger population, $\mathrm{HBV}$ infection remains endemic in those over 40 years of age [5].

As a consequence, many CRC patients in Asia also have chronic hepatitis $\mathrm{B}(\mathrm{CHB})$ infection. It has not been clearly expounded that the effect of concomitant $\mathrm{CHB}$ infection on the risk of colorectal liver metastasis (CRLM). Quite a number of clinicians believe that CHB has a "protective effect". Although previous reports support their hypothesis, these studies were limited by small sample size, mixed types of chronic hepatitis and xenotherapy [6]. Our previous study showed that CHB-induced liver cirrhosis is anti-metastatic, but failed to prove that active $\mathrm{CHB}$, represented by positive Hepatitis $\mathrm{B}$ e antigen (HBeAg) is pro-metastatic [7]. This current study expands upon our experience with a larger sample size and hopes to further address this issue.

\section{Materials and Methods}

\section{Data Source}

We performed this retrospective, cross-sectional study of 7187 consecutive newly diagnosed CRC cases in three hospitals. The Ethics Committee of whole three hospitals permitted this study. All patients were hospitalized during the period between January 2010 and January 2016 and had pathological confirmation of their CRC.

Retrospective data collection involved demographic information such as age, gender and laboratory studies, containing the complete blood counts, liver function tests and HBV specific tests. Liver fibrosis/cirrhosis was measured and calculated by the indicator that aspartate aminotransferase (AST) to platelet ratio index (APRI).According to the American Joint Committee on Cancer (AJCC) staging, tumor characteristics, involving tumor size, differentiation (well, moderate or poor) and tumor (T) and nodal stage $(\mathrm{N})$, were also noted based on pathology reports when available, or otherwise on imaging reports.

The definition and diagnosis of synchronous colorectal liver metastasis (synCRLM) was same as our preceding report [7]. SynCRLM was defined as the simultaneous diagnosis of liver metastasis together with primary CRC; in brief, all patients with CRC underwent chest radiography and abdominal and pelvic ultrasonography (US). If these studies suggested possible pulmonary and/or hepatic lesion(s), computed tomography (CT) scan was performed to confirm the diagnosis. All liver metastases were verified with $\mathrm{CT}$ scan that was reviewed independently by two senior radiotherapists. SynCRLM was defined as the simultaneous diagnosis of liver metastasis together with primary CRC.

This study was divided all patients into different groups depending upon the status of $\mathrm{HBsAg}\left(\mathrm{HBsAg}^{+}\right.$ vs. $\mathrm{HBsAg}$ ) or $\mathrm{HBeAg}\left(\mathrm{HBeAg}^{+}\right.$vs. $\mathrm{HBeAg}-$ ). Baseline clinicopathological parameters and the prevalence of synCRLM were compared between these groups.

\section{Inclusion and exclusion criteria}

The inclusion criteria are as followings: (1) all pathological sections that were primary adenocarcinomas in the colon and rectum; (2) no treatment before admission; (3) diagnosed of CRC based on both the postoperative pathology and the electronic colonoscopy biopsy; (4) had relatively complete medical data records.

The exclusion criteria are as followings: (1) patients who have had other treatments, not newly diagnosed or treated; (2) without pathological diagnosis; (3) with other concomitant malignancies.

\section{Statistical analysis}

The prevalence of synCRLM in different groups was compared by chi-square $\left(\chi^{2}\right)$ test. Baseline categorical clinical parameters were compared by $\chi^{2}$ test, and numerical values were compared using Student's $t$ test. APRI were compared by Wilcoxon rank sum test. Significant risk factors for synCRLM were analyzed first by univariate logistic regression analysis and then by multivariate logistic regression analysis. Statistical analysis was performed by SPSS17.0.

\section{Results}

Patients' information about HBV infection and synCRLM prevalence are presented in Figure 1. In the entire cohort of 7187 CRC patients, 7110 patients had the information of HBsAg test, the prevalence of HBsAg positivity was $5.18 \% \quad(368 / 7110)$; Baseline clinicopathological parameters of the $\mathrm{HBsAg}^{+}$and HBsAg- cohorts are presented in Table 1. The mean age of the HBsAg- patients was significantly higher than that of the $\mathrm{HBsAg}^{+}$patients (mean $\pm \mathrm{SD}$, $60.69 \pm 12.81$ vs. $56.66 \pm 11.61$ years, $P=0.001$ ).

The mean carbohydrate antigen 19-9 (CA19-9) value in $\mathrm{HBsAg}^{+}$patients was also slightly higher than that in HBsAg- patients but achieved statistical significance (55.81 \pm 144.66 vs. $55.64 \pm 235.67, P=0.001$ ). No statistical differences were found among tumor size or stage, gender distribution and carcinoembryonic antigen (CEA). 
7187 CRC Patients

- $627 \operatorname{syn} C R L M(8.72 \%)$

$\begin{array}{ll}77 \text { w/o HBsAg info } & 7110 \text { with HBsAg info } \\ \cdot 8 \text { synCRLM } & \cdot 619 \text { synCRLM }(8.71 \%) \\ & \cdot 6074 \text { with HBeAg info }\end{array}$

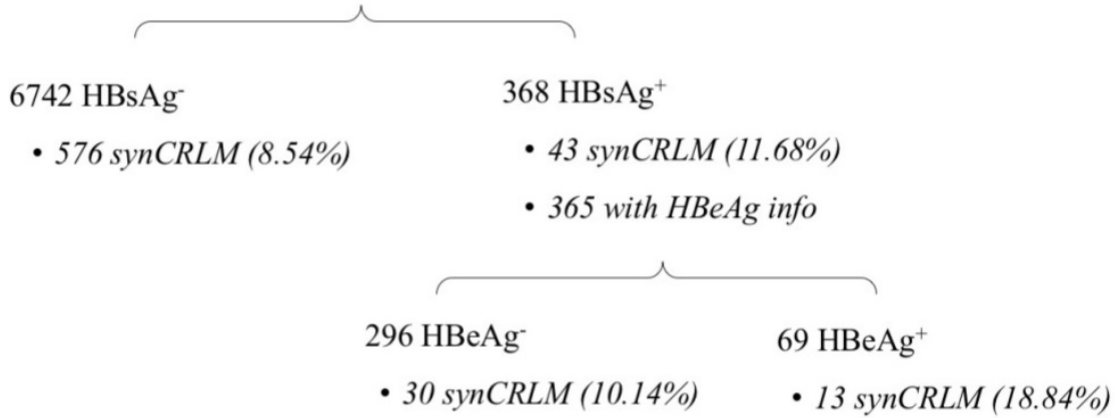

Figure 1. Number of patients with $\mathrm{HBs} \mathrm{Ag} / \mathrm{HBeAg}$ positivity and synCRLM prevalence.

Table 1. Baseline clinicopathological of $\mathrm{HBsAg}^{+}$and $\mathrm{HBsAg}$ cohorts

\begin{tabular}{|c|c|c|c|}
\hline Factors & $\mathrm{HBsAg}^{+}(\mathrm{N}=368)$ & HBsAg- $(\mathrm{N}=6742)$ & $P$ \\
\hline synCRLM (Yes/No) & $43 / 325$ & $576 / 6166$ & 0.037 \\
\hline Gender (male/female) & $214 / 154$ & $3975 / 2767$ & 0.759 \\
\hline Age (years, mean $\pm S D$ ) & $56.66 \pm 11.61$ & $60.69 \pm 12.81$ & 0.001 \\
\hline \multicolumn{4}{|l|}{ Initial manifestation } \\
\hline Bleeding or blood in stool & $93(25.27 \%)$ & $1702(25.24 \%)$ & \\
\hline $\begin{array}{l}\text { Change in the consistency, size or } \\
\text { shape of stools }\end{array}$ & $18(4.89 \%)$ & $275(4.08 \%)$ & \\
\hline $\begin{array}{l}\text { Change in frequency of bowel } \\
\text { movements }\end{array}$ & $11(2.99 \%)$ & $277(4.11 \%)$ & \\
\hline Abdominal pain or discomfort & $42(11.41 \%)$ & $1103(16.36 \%)$ & \\
\hline Abdominal mass & $2(0.54 \%)$ & $34(0.50 \%)$ & \\
\hline $\begin{array}{l}\text { N/A or other initial } \\
\text { manifestation }\end{array}$ & $202(54.90 \%)$ & $3345(49.62 \%)$ & \\
\hline $\begin{array}{l}\text { Asymptomatic colonoscopy exam } \\
\text { (ACE) }\end{array}$ & 0 & $6(0.09 \%)$ & 0.274 \\
\hline $\begin{array}{l}\text { synCRLM excluding ACE } \\
\text { (Yes/No) }\end{array}$ & $43 / 325$ & $576 / 6160$ & 0.038 \\
\hline \multicolumn{4}{|l|}{ Primary CRC } \\
\hline Tumor size $(\mathrm{cm})$ & $4.71 \pm 2.00$ & $4.74 \pm 2.04$ & 0.852 \\
\hline \multicolumn{4}{|l|}{ Location } \\
\hline Colon & $128(34.78 \%)$ & $2357(34.96 \%)$ & \\
\hline Rectum & $129(35.06 \%)$ & $2581(38.28 \%)$ & 0.516 \\
\hline $\mathrm{N} / \mathrm{A}$ & $111(30.16 \%)$ & $1804(26.76 \%)$ & \\
\hline \multicolumn{4}{|l|}{ Grade } \\
\hline Poorly & $59(16.03 \%)$ & $961(14.25 \%)$ & \\
\hline Moderately & $256(69.57 \%)$ & $4638(68.79 \%)$ & \\
\hline Well & $13(3.53 \%)$ & $235(3.49 \%)$ & 0.772 \\
\hline $\mathrm{N} / \mathrm{A}$ & $40(10.87 \%)$ & $908(13.47 \%)$ & \\
\hline \multicolumn{4}{|l|}{ T stage } \\
\hline Tis-T2 & $55(14.95 \%)$ & $1088(16.14 \%)$ & \\
\hline T3- T4 & $271(73.64 \%)$ & $4634(68.73 \%)$ & 0.336 \\
\hline $\mathrm{N} / \mathrm{A}$ & $42(11.41 \%)$ & $1020(15.13 \%)$ & \\
\hline \multicolumn{4}{|l|}{ N stage } \\
\hline No & $194(52.72 \%)$ & $3440(51.02 \%)$ & \\
\hline N1-N2 & $127(34.51 \%)$ & $2211(32.80 \%)$ & 0.876 \\
\hline $\mathrm{N} / \mathrm{A}$ & $47(12.77 \%)$ & $1091(16.18 \%)$ & \\
\hline CEA (ng/ml) & $28.21 \pm 109.65$ & $27.74 \pm 106.34$ & 0.557 \\
\hline CA199 $(\mathrm{U} / \mathrm{ml})$ & $55.81 \pm 144.66$ & $55.64 \pm 235.67$ & 0.001 \\
\hline
\end{tabular}

HBsAg: hepatitis B surface antigen; synCRLM: synchronous colorectal liver metastasis; N/A: not available; Tis: tumor in situ; CEA: Carcinoembryonic Antigen; CA199: Carbohydrate Antigen 19-9.
Complete blood counts and liver function parameters of the $\mathrm{HBsAg}^{+}$and $\mathrm{HBsAg}$ - cohorts are also presented in Table 1. White blood cell (WBC), neutrophil, eosinophils and platelet counts in $\mathrm{HBsAg}^{+}$patients were significantly lower than those of the HBsAg- patients, these can be explained by the CHB-related liver fibrosis/cirrhosis and hypersplenium, as the APRI value in the $\mathrm{HBsAg}^{+}$patients was significantly higher than in the HBsAg- patients ( $0.370 \pm 0.635$ vs. $0.225 \pm 0.477 ; P=0.001$; Table 1). Also, in the $\mathrm{HBsAg}^{+}$patients, their liver tests were significantly worse compared to the HBsAg- patients, indicated by the elevated aminotransferase, bilirubin and decreased globulin (GLB), prealbumin, triglyceride (TG), cholesterol (CHOL) and Low Density Lipoprotein (LDL).

The overall prevalence of synCRLM was $8.72 \%(627 / 7187)$. Moreover, the cohort of $\mathrm{HBsAg}^{+}$patients (43/368) was significantly higher than that of HBsAg- patients $(576 / 6742)(11.68 \%$ vs. $8.54 \%, P=0.037 ; \chi^{2}$ test; Table 1).

Based on the chief complains and the history of present illness in the medical record, the initial manifestations included bleeding or blood in stool; change in the consistency, size or shape of stools; change in frequency of bowel movements; abdominal pain or discomfort; abdominal mass. The most common initial manifestation was bleeding or blood in stool. Both $\mathrm{HBsAg}^{+}$and $\mathrm{HBsAg}$ - patients showed that no significant difference in the distribution of initial manifestation were observed $\left(P=0.274 ; \chi^{2}\right.$ test, Table 1). Only 6 out of 7187 patients were diagnosed by a routine asymptomatic colonoscopy examination, they were all HBsAg- and had no synCRLM; If these 6 
patients were excluded, synCRLM remained more prevalent in $\mathrm{HBsAg}^{+}$patients $(43 / 368)$ compared to HBsAg- patients (576/6736) $(11.68 \%$ vs. $8.55 \%, P=0$. 038; $\chi^{2}$ test, Table 1).

In 7110 patients with HBsAg information, 6074 patients had $\mathrm{HBeAg}$ test performed, and $\mathrm{HBeAg}$ positivity was noted in $1.14 \%$ patients $(69 / 6074)$. In $368 \mathrm{HBsAg}^{+}$patients, 365 patients also had HBeAg information. Baseline clinicopathological parameters of the $\mathrm{HBsAg}^{+} / \mathrm{HBeAg}^{+}$and $\mathrm{HBsAg}^{+} / \mathrm{HBeAg}^{-}$ cohorts are presented in Table 2. Male domination was significant in $\mathrm{HBeAg}^{+}$cohort $(69.57 \%$ vs. $55.74 \%$, $P=0.036)$. The CA19-9 value in $\mathrm{HBsAg}^{+} / \mathrm{HBeAg}^{+}$ patients was significantly higher than that in $\mathrm{HBsAg}^{+} / \mathrm{HBeAg}^{-}$patients

(100.22 221.79 vs. $45.17 \pm 117.24, \quad P=0.001)$. There were no significant differences in age, tumor size or stage and CEA.

Table 2. Complete blood counts of $\mathrm{HBsAg}^{+}$and $\mathrm{HBsAg}$ - cohorts

\begin{tabular}{llll}
\hline Factors & $\mathrm{HBsAg}^{+}(\mathrm{N}=368)$ & $\mathrm{HBsAg}^{-}(\mathrm{N}=6742)$ & $P$ \\
\hline Whole blood counts & & & \\
WBC $\left(10^{9} / \mathrm{L}\right)$ & $6.156 \pm 2.608$ & $6.564 \pm 2.790$ & $\mathbf{0 . 0 0 1}$ \\
Neutrophil $\left(10^{9} / \mathrm{L}\right)$ & $3.706 \pm 2.285$ & $4.060 \pm 2.299$ & $\mathbf{0 . 0 0 1}$ \\
Lymphocyte $\left(10^{9} / \mathrm{L}\right)$ & $1.714 \pm 0.774$ & $1.756 \pm 0.739$ & 0.191 \\
Monocyte $\left(10^{9} / \mathrm{L}\right)$ & $0.549 \pm 0.357$ & $0.566 \pm 0.365$ & 0.505 \\
Eosinophil $\left(10^{9} / \mathrm{L}\right)$ & $0.160 \pm 0.218$ & $0.165 \pm 0.329$ & $\mathbf{0 . 0 2 5}$ \\
Basophil $\left(10^{9} / \mathrm{L}\right)$ & $0.027 \pm 0.026$ & $0.029 \pm 0.338$ & 0.101 \\
RBC $\left(10^{12} / \mathrm{L}\right)$ & $4.261 \pm 0.585$ & $4.224 \pm 0.632$ & 0.388 \\
Platelet $\left(10^{9} / \mathrm{L}\right)$ & $217.213 \pm 83.052$ & $249.164 \pm 87.392$ & $\mathbf{0 . 0 0 1}$ \\
Hb $(\mathrm{g} / \mathrm{L})$ & $122.185 \pm 23.006$ & $120.333 \pm 24.010$ & 0.297 \\
\hline
\end{tabular}

WBC: white blood cell; RBC: red blood cell; $\mathrm{Hb}$ : hemoglobin.

Complete blood counts and liver function parameters of the $\mathrm{HBsAg}^{+} / \mathrm{HBeAg}^{+}$and $\mathrm{HBsAg}^{+}$/ $\mathrm{HBeAg}$ - cohorts are also presented in Table 2. Here only neutrophil and platelet counts were significantly lower in the $\mathrm{HBsAg}^{+} / \mathrm{HBeAg}^{+}$patients compared with $\mathrm{HBsAg}^{+} / \mathrm{HBeAg}$ patients, and double positive patients did have significantly higher APRI value as well as aminotransferase, GGT and ALP, but not bilirubin. These results indicated that the $\mathrm{HBsAg}^{+} /$ $\mathrm{HBeAg}^{+}$cohort is in general more fibrotic or cirrhotic.

In $365 \mathrm{HBsAg}^{+}$patients with $\mathrm{HBeAg}$ information, $19 \mathrm{HBsAg}^{+} / \mathrm{HBeAg}^{+}$patients and 40 $\mathrm{HBsAg}^{+} / \mathrm{HBeAg}$ patients showed elevated ALT, while only 2 of them were under the anti-HBV treatment. There were other 7 patients with normal ALT are under or had a history of anti-HBV treatment. The anti-HBV treatments includes: 1 with interferon-a, 1 with lamivudine, 2 with adefovir, 2 with lamivudine plus adefovir and 3 with entecavir.

In further sub-group analysis, synCRLM was also more prevalent in $\mathrm{HBsAg}^{+} / \mathrm{HBeAg}^{+}$patients $(13 / 69)$ compared with $\mathrm{HBsAg}^{+} / \mathrm{HBeAg}$ patients (30/296) (18.84\% vs. $10.14 \%, P=0.043 ; \chi^{2}$ test, Table 2$)$. If 9 patients with anti-HBV treatment were excluded,
synCRLM remained more prevalent in $\mathrm{HBsAg}^{+}$/ $\mathrm{HBeAg}^{+}$patients $(13 / 67)$ compared to $\mathrm{HBsAg}^{+} /$ HBeAg- patients (30/289) (19.40\% vs. $10.38 \%, P=0.032$; $\chi^{2}$ test, Table 2).

In univariate logistic regression analysis, the odds ratio (OR) of $\mathrm{HBeAg}$ positivity was the highest [OR: 2.920, 95\% confidence interval (CI): 1.588-5.371, $P=0.001$; Table 3], which was more than twice that of HBsAg positivity (OR: 1.417, 95\% CI: 1.019-1.969, $P=0.038$; Table 3 ). In the subsequent multivariate analysis with other significant factors, $\mathrm{HBeAg}$ positivity remained the second strongest predictor of synCRLM only after CEA. (OR: 2.622, 95\% CI: 1.164-5.903, $P=0.020$; Table 4), the OR of HBsAg positivity was 1.565 (95\% CI: 1.009-2.427, $P=0.046$; Table 5).

Table 3. Liver function parameters of $\mathrm{HBsAg}^{+}$and $\mathrm{HBsAg}$ cohorts

\begin{tabular}{lllc}
\hline Factors & $\mathrm{HBsAg}^{+}(\mathrm{N}=368)$ & HBsAg- $(\mathrm{N}=6742)$ & $P$ \\
\hline Liver function parameters & & & \\
ALT (U/L) & $24.782 \pm 28.464$ & $17.436 \pm 15.525$ & $\mathbf{0 . 0 0 1}$ \\
AST (U/L) & $24.221 \pm 16.396$ & $19.627 \pm 13.550$ & $\mathbf{0 . 0 0 1}$ \\
TBIL (umol/L) & $12.349 \pm 6.554$ & $11.191 \pm 12.145$ & $\mathbf{0 . 0 0 1}$ \\
DBIL (umol/L) & $4.227 \pm 2.481$ & $4.017 \pm 3.103$ & $\mathbf{0 . 0 4 1}$ \\
IBIL (umol/L) & $8.187 \pm 4.921$ & $7.143 \pm 4.449$ & $\mathbf{0 . 0 0 1}$ \\
GGT (U/L) & $25.919 \pm 35.446$ & $26.153 \pm 52.675$ & 0.393 \\
ALP (U/L) & $73.682 \pm 22.497$ & $75.131 \pm 38.434$ & 0.511 \\
ALB (g/L) & $38.393 \pm 5.160$ & $38.415 \pm 5.098$ & 0.964 \\
GLB (g/L) & $13.495 \pm 13.206$ & $26.440 \pm 6.735$ & $\mathbf{0 . 0 0 1}$ \\
Prealbumin (mg/L) & $191.311 \pm 61.890$ & $210.576 \pm 77.299$ & $\mathbf{0 . 0 0 1}$ \\
TG (mmol/L) & $1.041 \pm 0.477$ & $1.230 \pm 0.769$ & $\mathbf{0 . 0 0 1}$ \\
CHOL (mmol/L) & $4.461 \pm 1.060$ & $4.632 \pm 1.123$ & $\mathbf{0 . 0 1 1}$ \\
TBA (umol/L) & $6.933 \pm 7.871$ & $4.967 \pm 5.376$ & $\mathbf{0 . 0 0 1}$ \\
HDL (mmol/L) & $1.240 \pm 0.356$ & $1.247 \pm 1.252$ & 0.69 \\
LDL (mmol/L) & $2.647 \pm 0.856$ & $2.845 \pm 3.775$ & $\mathbf{0 . 0 0 4}$ \\
APRI & $0.370 \pm 0.635$ & $0.225 \pm 0.477$ & $\mathbf{0 . 0 0 1}$ \\
\hline TBIL: Total Bilirubin; DBIL: Direct Bilirubin; IBIL: Indirect Bilirubin; ALT: Alanine \\
Transaminase; AST: Aspartate Transaminase; GGT:Gamma-glutamyltransferase; \\
ALP: Alkaline Phosphatase; ALB: albumin; GLB: globulin; TG: triglyceride; CHOL: \\
cholesterol; TBA: Total Bile Acids; HDL: High Density Lipoprotein; LDL: Low \\
Density Lipoprotein; APRI: Aspartate Aminotransferase-to-platelet Ratio Index. \\
\hline
\end{tabular}

\section{Discussion}

In our previous study of 4033 CRC patients, contrary to much of the reported literature, positive HBsAg was associated with a significant increase in the prevalence of CRLM. Patients with active viral replication as determined by HBeAg positivity also trended toward increased CRLM prevalence but did not quite reach statistical 'significance [8]. In this study, we used the same criteria to recruit 3154 patients from the First Affiliated Hospital of Zhengzhou University as a third cohort. $\mathrm{HBsAg}+/ \mathrm{HBeAg}^{+}$patients showed significantly higher APRI value, AST, GGT and ALP as well as decreased neutrophil and platelet counts, suggesting that they were generally more fibrotic compared to $\mathrm{HBsAg}^{+} / \mathrm{HBeAg}^{-}$patients. Our 
previous study demonstrated that CHB-induced liver fibrosis/cirrhosis was anti-metastatic, as an increased APRI was associated with lower risk of CRLM [9]. Despite this, $\mathrm{HBsAg}^{+} / \mathrm{HBeAg}^{+}$patients had significantly higher prevalence of synCRLM. By univariate and multivariate logistic regression analysis, $\mathrm{HBeAg}$ positivity was an independent predictor of CRLM with higher odds ratio than HBsAg positivity.

Table 4. Baseline clinicopathological of $\mathrm{HBsAg}^{+} / \mathrm{HBeAg}^{+}$and $\mathrm{HBsAg}^{+} / \mathrm{HBeAg}^{-}$cohorts

\begin{tabular}{|c|c|c|c|}
\hline Factors & $\begin{array}{l}\mathrm{HBsAg}^{+} / \mathrm{HBeAg}^{+} \\
(\mathrm{N}=69)\end{array}$ & $\begin{array}{l}\mathrm{HBsAg}^{+} / \mathrm{HBeAg}^{-} \\
(\mathrm{N}=296)\end{array}$ & $P$ \\
\hline synCRLM (Yes/No) & $13 / 56$ & $30 / 266$ & 0.043 \\
\hline Gender (male/female) & $48 / 21$ & $165 / 131$ & 0.036 \\
\hline Age (years, mean $\pm S D$ ) & $54.16 \pm 12.71$ & $57.31 \pm 11.32$ & 0.066 \\
\hline \multicolumn{4}{|l|}{ Anti-HBV treatment } \\
\hline with & 2 & 7 & \\
\hline synCRLM (Yes/No) & $0 / 2$ & $1 / 6$ & 0.571 \\
\hline $\mathrm{W} / \mathrm{o}$ & 67 & 289 & \\
\hline synCRLM (Yes/No) & $13 / 54$ & $29 / 260$ & 0.032 \\
\hline \multicolumn{4}{|l|}{ Primary CRC } \\
\hline Tumor size $(\mathrm{cm})$ & $4.43 \pm 2.27$ & $4.78 \pm 1.94$ & 0.100 \\
\hline \multicolumn{4}{|l|}{ Location } \\
\hline Colon & $27(39.13 \%)$ & $99(33.45 \%)$ & \\
\hline Rectum & $20(28.99 \%)$ & $108(36.49 \%)$ & 0.234 \\
\hline N/A & $22(31.88 \%)$ & $89(30.06 \%)$ & \\
\hline \multicolumn{4}{|l|}{ Grade } \\
\hline Poorly & $11(15.94 \%)$ & $48(16.22 \%)$ & \\
\hline Moderately & $50(72.46 \%)$ & $204(68.92 \%)$ & \\
\hline Well & $3(4.35 \%)$ & $10(3.38 \%)$ & 0.935 \\
\hline $\mathrm{N} / \mathrm{A}$ & $5(7.25 \%)$ & $34(11.49 \%)$ & \\
\hline \multicolumn{4}{|l|}{ T stage } \\
\hline Tis-T2 & $10(14.49 \%)$ & $44(14.86 \%)$ & \\
\hline T3- T4 & $54(78.26 \%)$ & $215(72.64 \%)$ & 0.069 \\
\hline $\mathrm{N} / \mathrm{A}$ & $5(7.25 \%)$ & $37(12.50 \%)$ & \\
\hline \multicolumn{4}{|l|}{ N stage } \\
\hline No & $39(56.52 \%)$ & $153(51.69 \%)$ & \\
\hline N1-N2 & $24(34.78 \%)$ & $102(34.46 \%)$ & 0.077 \\
\hline N/A & $6(8.70 \%)$ & $41(13.85 \%)$ & \\
\hline CEA & $17.47 \pm 36.62$ & $31.09 \pm 121.36$ & 0.894 \\
\hline CA199 & $100.22 \pm 221.79$ & $45.17 \pm 117.24$ & 0.001 \\
\hline
\end{tabular}

HBsAg: hepatitis B surface antigen; synCRLM: synchronous colorectal liver metastasis; N/A: not available; Tis: tumor in situ; CEA: Carcinoembryonic Antigen; CA199: Carbohydrate Antigen 19-9.

According to the AASLD (American Association for the Study of Liver Diseases) 2018 Hepatitis B Guidance, CHB patients with elevated ALT should be evaluated with other tests (HBV-DNA, HBeAg, etc.) to determine the need for treatment with antiviral agents [10]. In our study, $59 \mathrm{HBsAg}^{+}$patients showed elevated ALT, but only 2 of them received anti-HBV treatment. In China, only $19 \%$ of $\mathrm{CHB}$ patients are diagnosed and only $10-11 \%$ of eligible $\mathrm{CHB}$ patients receive anti-HBV treatment [11]. Only very limited number of $\mathrm{CHB}$ patients in our study received or are currently receiving anti-HBV treatment, excluding these patients from the analysis would not affect the results and synCRLM remained more prevalent in
$\mathrm{HBsAg}^{+} / \mathrm{HBeAg}^{+}$patients. Future studies with a larger proportion of treated patients separately analyzed would be needed to observe the true effect of antiviral agents on the risk of CRLM.

Table 5. Complete blood counts of $\mathrm{HBsAg}^{+} / \mathrm{HBeAg}^{+}$and $\mathrm{HBsAg}^{+} / \mathrm{HBeAg}^{-}$cohorts

\begin{tabular}{llll}
\hline Factors & $\begin{array}{l}\mathrm{HBsAg}^{+} / \mathrm{HBeAg}^{+} \\
(\mathrm{N}=69)\end{array}$ & $\begin{array}{l}\mathrm{HBsAg}^{+} / \mathrm{HBeAg}^{-} \\
(\mathrm{N}=296)\end{array}$ & $P$ \\
\hline Complete blood counts & & & \\
WBC $\left(10^{9} / \mathrm{L}\right)$ & $5.531 \pm 1.621$ & $6.290 \pm 2.733$ & 0.087 \\
Neutrophil $\left(10^{9} / \mathrm{L}\right)$ & $3.097 \pm 1.386$ & $3.833 \pm 2.404$ & $\mathbf{0 . 0 1 3}$ \\
Lymphocyte $\left(10^{9} / \mathrm{L}\right)$ & $1.757 \pm 0.653$ & $1.709 \pm 0.796$ & 0.375 \\
Monocyte $\left(10^{9} / \mathrm{L}\right)$ & $0.494 \pm 0.222$ & $0.581 \pm 0.385$ & 0.601 \\
Eosinophil $\left(10^{9} / \mathrm{L}\right)$ & $0.161 \pm 0.121$ & $0.160 \pm 0.234$ & 0.071 \\
Basophil $\left(10^{9} / \mathrm{L}\right)$ & $0.027 \pm 0.019$ & $0.027 \pm 0.027$ & 0.349 \\
RBC $\left(10^{12} / \mathrm{L}\right)$ & $4.246 \pm 0.502$ & $4.265 \pm 0.601$ & 0.979 \\
Platelet $\left(10^{9} / \mathrm{L}\right)$ & $192.507 \pm 96.747$ & $222.932 \pm 77.912$ & $\mathbf{0 . 0 0 2}$ \\
Hb $(\mathrm{g} / \mathrm{L})$ & $126.962 \pm 22.316$ & $121.156 \pm 23.101$ & 0.071 \\
\hline
\end{tabular}

WBC: white blood cell; RBC: red blood cell; Hb: hemoglobin.

Table 6. Liver function parameters of $\mathrm{HBsAg} / \mathrm{HBeAg}^{+}$and $\mathrm{HBsAg}+/ \mathrm{HBeAg}-$ cohorts

\begin{tabular}{|c|c|c|c|}
\hline Factors & $\begin{array}{l}\mathrm{HBsAg}^{+} / \mathrm{HBeAg}^{+} \\
(\mathrm{N}=69)\end{array}$ & $\begin{array}{l}\mathrm{HBsAg}^{+} / \mathrm{HBeAg}^{-} \\
(\mathrm{N}=296)\end{array}$ & $P$ \\
\hline \multicolumn{4}{|l|}{ ALT elevation } \\
\hline ALT $>$ ULN but $<2 \times$ ULN & 16 & 30 & 0.425 \\
\hline $\mathrm{ALT} \geq 2 \times \mathrm{ULN}$ & 3 & 10 & \\
\hline \multicolumn{4}{|l|}{ Liver function } \\
\hline $\operatorname{ALB}(\mathrm{g} / \mathrm{L})$ & $37.429 \pm 5.566$ & $38.605 \pm 5.056$ & 0.095 \\
\hline GLB (g/L) & $12.705 \pm 13.565$ & $13.540 \pm 13.129$ & 0.622 \\
\hline Prealbum $(\mathrm{mg} / \mathrm{L})$ & $178.213 \pm 58.853$ & $193.847 \pm 62.448$ & 0.110 \\
\hline $\mathrm{TG}(\mathrm{mmol} / \mathrm{L})$ & $0.937 \pm 0.250$ & $1.064 \pm 0.511$ & 0.470 \\
\hline $\mathrm{CHOL}(\mathrm{mmol} / \mathrm{L})$ & $4.653 \pm 1.262$ & $4.422 \pm 1.014$ & 0.206 \\
\hline TBA (umol/L) & $8.181 \pm 9.225$ & $6.696 \pm 7.605$ & 0.279 \\
\hline $\mathrm{HDL}(\mathrm{mmol} / \mathrm{L})$ & $1.203 \pm 0.306$ & $1.247 \pm 0.366$ & 0.789 \\
\hline $\mathrm{LDL}(\mathrm{mmol} / \mathrm{L})$ & $2.844 \pm 1.128$ & $2.608 \pm 0.788$ & 0.335 \\
\hline APRI & $0.684 \pm 1.314$ & $0.294 \pm 0.264$ & 0.001 \\
\hline
\end{tabular}

Although the pathogenesis of how HBV influences CRC is not completely clear, there are potential mechanisms that should be considered. Virologists have discovered that active HBV replication raised the expression of a group of chemokines, including CCL20, CXCL6 and the CXCL9/10/ 11 family. Notably, these chemokines showed a stepwise increase from healthy individuals to asymptomatic $\mathrm{HBV}$ carriers and then to patients with CHB [12]. Oncologists and gastroenterologists have developed substantial evidence that these same chemokines increase the risk of CRLM [13]. To our knowledge, no study has linked these concepts to propose a mechanism for CRLM pathogenesis in the face of HBV. We are currently exploring this further by performing chemokine screening on CRLM 
patients to better delineate the relevant chemokines and their mechanisms.

Table 7. Univariate logistic regression analysis of the significant predictors for synCRLM

\begin{tabular}{|c|c|c|c|c|c|c|}
\hline Variable & Coefficient & SE & Wald $x 2$ & $P$ & $\begin{array}{l}\text { Odds } \\
\text { ratio }\end{array}$ & $95 \% \mathrm{CI}$ \\
\hline Gender & -0.223 & 0.087 & 6.597 & $0.010^{*}$ & 0.800 & $0.675-0.949$ \\
\hline Age & -0.132 & 0.084 & 2.485 & 0.115 & 0.876 & $0.743-1.033$ \\
\hline Glu & -0.074 & 0.087 & 0.730 & 0.393 & 0.928 & $0.782-1.101$ \\
\hline HBsAg & 0.348 & 0.168 & 4.296 & $0.038^{*}$ & 1.417 & $1.019-1.969$ \\
\hline HBeAg & 1.072 & 0.311 & 11.878 & $0.001^{*}$ & 2.920 & $1.588-5.371$ \\
\hline WBC & 0.461 & 0.088 & 27.522 & $<0.001^{*}$ & 1.585 & $1.334-1.883$ \\
\hline Neutrophil & 0.668 & 0.090 & 55.371 & $<0.001^{*}$ & 1.950 & $1.636-2.325$ \\
\hline Lymphocyte & -0.273 & 0.087 & 9.788 & $0.002^{*}$ & 0.761 & $0.642-0.903$ \\
\hline Monocyte & 0.519 & 0.088 & 34.513 & $<0.001^{*}$ & 1.680 & $1.413-1.997$ \\
\hline Eosinophils & 0.188 & 0.086 & 4.753 & $0.029 *$ & 1.207 & $1.019-1.430$ \\
\hline Basophils & -0.186 & 0.087 & 4.580 & $0.032^{*}$ & 0.830 & $0.700-0.984$ \\
\hline $\mathrm{RBC}$ & -0.101 & 0.086 & 1.378 & 0.240 & 0.904 & $0.763-1.070$ \\
\hline $\mathrm{Hb}$ & -0.185 & 0.087 & 4.527 & $0.033^{*}$ & 0.831 & $0.701-0.986$ \\
\hline Platelet & 0.195 & 0.086 & 5.158 & $0.023^{*}$ & 1.215 & $1.027-1.437$ \\
\hline Neu_pct & 0.671 & 0.090 & 55.496 & $<0.001^{*}$ & 1.957 & $1.640-2.335$ \\
\hline Lym_pct & -0.813 & 0.092 & 78.365 & $<0.001^{*}$ & 0.443 & $0.370-0.531$ \\
\hline Mono_pct & 0.259 & 0.087 & 8.924 & $0.003^{*}$ & 1.295 & $1.093-1.535$ \\
\hline Eosi_pct & -0.003 & 0.086 & 0.001 & 0.970 & 0.997 & $0.842-1.180$ \\
\hline Baso_pct & -0.196 & 0.089 & 4.838 & $0.028^{*}$ & 0.822 & $0.691-0.979$ \\
\hline ALT & 0.561 & 0.088 & 40.644 & $<0.001^{*}$ & 1.753 & $1.457-2.083$ \\
\hline AST & 0.876 & 0.089 & 97.180 & $<0.001^{*}$ & 2.402 & $2.018-2.859$ \\
\hline GGT & 1.180 & 0.097 & 149.362 & $<0.001^{*}$ & 3.254 & 2.693-3.932 \\
\hline ALP & 0.789 & 0.090 & 76.963 & $<0.001^{*}$ & 2.201 & $1.846-2.626$ \\
\hline TBIL & 0.014 & 0.086 & 0.026 & 0.871 & 1.014 & 0.857-1.199 \\
\hline DBIL & -0.039 & 0.088 & 0.197 & 0.657 & 0.962 & $0.809-1.143$ \\
\hline IBIL & 0.019 & 0.086 & 0.050 & 0.823 & 1.019 & $0.861-1.206$ \\
\hline HDL & -0.200 & 0.104 & 3.723 & 0.054 & 0.818 & $0.668-1.003$ \\
\hline LDL & 0.374 & 0.105 & 11.012 & $0.001^{*}$ & 1.415 & $1.153-1.736$ \\
\hline Prealbumin & -0.472 & 0.092 & 26.328 & $<0.001^{*}$ & 0.624 & $0.521-0.747$ \\
\hline ALB & -0.289 & 0.087 & 11.163 & $0.001^{*}$ & 0.749 & $0.632-0.887$ \\
\hline GLB & 0.221 & 0.086 & 6.616 & $0.010^{*}$ & 1.248 & $1.054-1.477$ \\
\hline TG & -0.288 & 0.104 & 7.678 & $0.006^{*}$ & 0.750 & $0.612-0.919$ \\
\hline CHOL & 0.230 & 0.103 & 4.970 & $0.026^{*}$ & 1.259 & $1.028-1.542$ \\
\hline TBA & 0.105 & 0.095 & 1.214 & 0.271 & 1.111 & 0.921-1.339 \\
\hline LDH & 1.071 & 0.128 & 63.407 & $<0.001^{*}$ & 2.764 & $2.152-3.549$ \\
\hline NLR & 0.709 & 0.090 & 61.497 & $<0.001^{*}$ & 2.032 & $1.702-2.426$ \\
\hline CEA & 1.795 & 0.118 & 229.703 & $<0.001^{*}$ & 6.018 & $4.772-7.590$ \\
\hline CA199 & 1.281 & 0.104 & 153.030 & $<0.001^{*}$ & 3.601 & $2.939-4.441$ \\
\hline Size & 0.143 & 0.112 & 1.638 & 0.201 & 1.154 & $0.927-1.437$ \\
\hline Location & -0.083 & 0.038 & 4.679 & $0.031^{*}$ & 0.921 & 0.854-0.992 \\
\hline Differentiated & -0.214 & 0.122 & 3.073 & 0.080 & 0.807 & $0.636-1.026$ \\
\hline $\mathrm{T}$ & 0.511 & 0.079 & 42.130 & $<0.001^{*}$ & 1.667 & $1.428-1.945$ \\
\hline $\mathrm{N}$ & 0.417 & 0.063 & 43.897 & $<0.001^{*}$ & 1.518 & $1.342-1.717$ \\
\hline M & 2.310 & 0.091 & 638.946 & $<0.001^{*}$ & 10.074 & $8.422-12.050$ \\
\hline
\end{tabular}

GLU: glucose; HBsAg: hepatitis B surface antigen;HBeAg: hepatitis B e antigen; WBC: white blood cell; RBC: red blood cell; Hb: hemoglobin; Neu_pct: Neutrophil percentage; Lym_pct: Lymphocyte percentage; Mono_pct: Monocyte percentage; Eosi_pct: Eosinophils percentge; Baso_pct: Basophils percentage; ALT: Alanine Transaminase; AST: Aspartate Transaminase; GGT: Gamma-glutamyltransferase; ALP: Alkaline Phosphatase; TBIL: Total Bilirubin; DBIL: Direct Bilirubin; IBIL: Indirect Bilirubin; HDL: High Density Lipoprotein; LDL: Low Density Lipoprotein; ALB: albumin; GLB: globulin; TG: triglyceride; CHOL: cholesterol; TBA: Total Bile Acids; LDH: Lactate Dehydrogenase; NLR: Neutrophil to Lymphocyte Ratio; CEA: Carcinoembryonic Antigen; CA199: Carbohydrate Antigen 199.

Unlike the minimal chance of becoming HBsAg negative, HBV-DNA suppression and the loss of HBeAg can be achieved in many patients with $\mathrm{CHB}$ using current anti-viral therapies [14]. HBeAg may be a more useful marker in CRC patients. If reduction in viral replication and infectivity can be achieved with anti-viral agents in patients, this may be potentially beneficial for decreasing CRLM and prolonging life in CRC patients who also have HBV.

Table 8. Multivariate logistic regression analysis of the predictors for synCRLM with $\mathrm{HBeAg}$

\begin{tabular}{lllllll}
\hline Variable & Coefficient & SE & \multicolumn{2}{l}{ Wald $x \mathbf{2}$} & $P$ & \multicolumn{2}{l}{ Odds ratio $95 \%$ CI } \\
\hline HBeAg & $\mathbf{0 . 9 6 4}$ & $\mathbf{0 . 4 1 4}$ & $\mathbf{5 . 4 1 6}$ & $\mathbf{0 . 0 2 0}$ & $\mathbf{2 . 6 2 2}$ & $\mathbf{1 . 1 6 4}$ to $\mathbf{5 . 9 0 3}$ \\
CEA & 1.110 & 0.152 & 53.258 & $<0.001$ & 3.034 & 2.252 to 4.087 \\
ALT & 0.242 & 0.144 & 2.828 & 0.093 & 1.274 & 0.961 to 1.688 \\
GGT & 0.681 & 0.147 & 21.455 & $<0.001$ & 1.975 & 1.481 to 2.635 \\
Platelet & 0.320 & 0.137 & 5.488 & 0.019 & 1.378 & 1.054 to 1.801 \\
T & 0.221 & 0.112 & 3.897 & 0.048 & 1.248 & 1.002 to 1.554 \\
N & 0.001 & 0.091 & 0.000 & 0.995 & 1.001 & 0.837 to 1.196 \\
M & 2.297 & 0.145 & 250.733 & $<0.001$ & 9.941 & 7.481 to 13.209 \\
Constant & -5.325 & 0.383 & 193.781 & 0.000 & 0.005 & \\
\hline
\end{tabular}

synCRLM: synchronous colorectal liver metastasis; HBeAg: hepatitis B e antigen; CEA: Carcinoembryonic Antigen; GGT: Gamma-glutamyltransferase; PLT: platelet; ALT: Alanine Transaminase; SE: Standard error; $95 \%$ CI: $95 \%$ confidence interval.

Table 9. Multivariate logistic regression analysis of the predictors for synCRLM with $\mathrm{HBsAg}$

\begin{tabular}{lllllll}
\hline Variable & Coefficient & SE & Wald $x 2$ & $P$ & \multicolumn{2}{l}{ Odds ratio $95 \%$ CI } \\
\hline HBsAg & $\mathbf{0 . 4 4 8}$ & $\mathbf{0 . 2 2 4}$ & $\mathbf{3 . 9 9 5}$ & $\mathbf{0 . 0 4 6}$ & $\mathbf{1 . 5 6 5}$ & $\mathbf{1 . 0 0 9}$ to $\mathbf{2 . 4 2 7}$ \\
CEA & 1.357 & 0.140 & 93.580 & $<0.001$ & 3.885 & 2.951 to 5.114 \\
ALT & 0.281 & 0.127 & 4.917 & 0.027 & 1.325 & 1.033 to 1.698 \\
GGT & 0.761 & 0.130 & 34.463 & $<0.001$ & 2.140 & 1.660 to 2.759 \\
Platelet & 0.325 & 0.120 & 7.293 & 0.007 & 1.383 & 1.093 to 1.751 \\
T & 0.274 & 0.097 & 7.930 & 0.005 & 1.316 & 1.087 to 1.593 \\
N & 0.110 & 0.077 & 2.026 & 0.155 & 1.116 & 0.959 to 1.299 \\
M & 2.048 & 0.130 & 249.717 & $<0.001$ & 7.753 & 6.014 to 9.995 \\
Constant & -5.591 & 0.339 & 272.254 & 0.000 & 0.005 &
\end{tabular}

synCRLM: synchronous colorectal liver metastasis; HBsAg: hepatitis B surface antigen; CEA: Carcinoembryonic Antigen; ALT: Alanine Transaminase; GGT:

Gamma-glutamyltransferase; SE: Standard error; 95\%CI: 95\% confidence interval.

This study is limited in that it is a retrospective cross-sectional prevalence study; however it would be impossible to study synCRLM in a prospective manner. A prospective study of all CRC patients to observe for metachronous CRLM stratified for HBV and with measurement of various chemokines would be a theoretical way to understand this. However, such a study would be practically too complex to conduct because it would require long-term follow up and the identical parallel use of neoadjuvant and adjuvant chemotherapy, newer targeted therapy and immunotherapy plus the use of antivirals for $\mathrm{HBV}$. There would be too many variables which may affect each other to understand the true effect of HBV on development of CRLM.

Screening colonoscopy can reduce the incidence of CRC by identifying and removing precancerous polyps and by prompting an increase in the surveillance frequency. Unfortunately, China does not currently have a nationwide CRC screening or surveillance programs with colonoscopy. Awareness 
and education on CRC in most of China is generally poor, only very limited individuals with better education and socioeconomic status privately pay for the screening colonoscopy examinations. Even among high-risk populations of CRC in urban China, where the education is considered to be better than rural areas, the compliance rate for freely-provided colonoscopy was only $15.3 \%$ [15].

China's hospitals do not have centralized electronic medical record system, instead, every hospital use its own hospital information system (HIS) and medical record system (MRS). As the consequence, we can't check whether these patients had previous colonoscopy examinations or not. Based on the chief complains and the history of present illness in the current medical record, we meticulously checked the initial manifestation of all 7187 patients, and discover that only 6 patients were diagnosed by a routine asymptomatic colonoscopy examination, exclusion of these 6 patients does not affect the statistic results of the prevalence between $\mathrm{HBsAg}^{+}$ and HBsAg- cohorts. Therefore, it is unlikely that the use of screening colonoscopy presented a potential bias between the groups.

As the burden of CRC continues to rise in countries in which $\mathrm{CHB}$ is endemic, serologic screening for $\mathrm{CHB}$ in newly diagnosed $\mathrm{CRC}$ should be advised. Screening should also be considered in non-endemic areas if patients have risk factors for $\mathrm{HBV}$ and their viral status is not known. Antiviral agents are typically given to prevent the dangerous consequences of $\mathrm{HBV}$ reactivation while on chemotherapy. Patients who have relatively advanced CRC (stage IIb or higher) will likely receive neoadjuvant or adjuvant chemotherapy and those patients who also have concomitant $\mathrm{CHB}$ should have anti-HBV therapy initiated. However, as CRC screening programs have increased the detection of early stage CRC patients [16], it will be important to develop strategies for prevention of CRC progression. Early stage CRC patients may undergo only surgical resection but are still at risk for developing CRLM in the future. The 5-year cumulative metachronous CRLM rate was reported to reach $3.7 \%$ and $13.3 \%$ for TNM stage I and II CRC, respectively [17]. If the association between active $\mathrm{CHB}$ and the risk of CRLM is validated with other studies, it will be essential to determine the optimal candidate for HBV therapy in terms of liver function and stage of fibrosis/cirrhosis, the timing/length of therapy and surveillance for CRLM.

This study has limitations. First, we lacked HBV information of some patients, so the missing data may cause information bias. Then, the sample size is limited, and the research method is vulnerable to the influence of unbalanced quality method. Finally, we believe that future research can continue to focus on the prevention and efficacy of CRLM after antiviral clinical treatment.

\section{Conclusions}

In summary, $\mathrm{HBeAg}$ positivity is a clinical risk factor for CRLM that can be readily identified and disposed. It is yet unclear if antiviral treatment can decrease the risk of liver metastasis in CRC patients, but future studies with carefully designed prospective trials will be needed to better define this.

\section{Abbreviations}

CRC: colorectal cancer; $\mathrm{CHB}$ : chronic hepatitis B; CRLM: colorectal liver metastasis; HBeAg: hepatitis B surface antigen; HBeAg: hepatitis B e antigen; HBV: hepatitis B virus; synCRLM: synchronous colorectal liver metastasis; AST: aspartate aminotransferase; APRI: aspartate aminotransferase to platelet ratio index; CEA: carcinoembryonic antigen; CA19-9: carbohydrate antigen 19-9; WBC: White blood cell; GLB: globulin; TG: triglyceride; CHOL: cholesterol; LDL: Low Density Lipoprotein; GGT: Gammaglutamyltransferase; ALP: Alkaline Phosphatase.

\section{Acknowledgements}

LZ is funded by the National Natural Science Fund of China (No. 81272375 and No. 81872400).

\section{Ethical approval and consent to participate}

The study was approved by the Ethics Committee of First Affiliated Hospital of Zhengzhou University and the Ethics Committee of the Affiliated Hospital of Qingdao University, with waiver of informed consent. There are two reasons. First, the time span of this study is long. Second, no patient privacy information is involved.

\section{Competing Interests}

LZ is on the speakers' bureau for Bayer and MSD; JC and LLW are on the speakers' bureau for Bayer; others declare that they have no conflict of interest.

\section{References}

1. Feng RM, Zong YN, Cao SM, Xu RH. Current cancer situation in China: good or bad news from the 2018 Global Cancer Statistics? Cancer Commun (Lond). 2019; 39: 22.

2. Zhao L. Advances in chronic liver diseases with abnormal chemokine expression and colorectal cancer liver metastasis. Chin J Clin Oncol. 2020; 47: 552-6.

3. Polaris Observatory C. Global prevalence, treatment, and prevention of hepatitis B virus infection in 2016: a modelling study. Lancet Gastroenterol Hepatol. 2018; 3: 383-403.

4. Cui F, Shen L, Li L, Wang H, Wang F, Bi S, et al. Prevention of Chronic Hepatitis B after 3 Decades of Escalating Vaccination Policy, China. Emerg Infect Dis. 2017; 23: 765-72. 
5. Li Destri G, Castaing M, Ferlito F, Minutolo V, Di Cataldo A, Puleo S. Rare hepatic metastases of colorectal cancer in livers with symptomatic HBV and HCV hepatitis. Ann Ital Chir. 2013; 84: 323-7.

6. Wang FS, Shao ZG, Zhang JL, Liu YF. Colorectal liver metastases rarely occur in patients with chronic hepatitis virus infection. Hepatogastroenterology. 2012; 59: 1390-2.

7. Qiu HB, Zhang LY, Zeng ZL, Wang ZQ, Luo HY, Keshari RP, et al. HBV infection decreases risk of liver metastasis in patients with colorectal cancer: A cohort study. World J Gastroenterol. 2011; 17: 804-8.

8. Huo T, Cao J, Tian Y, Shi X, Wu L, Zhang M, et al. Effect of Concomitant Positive Hepatitis B Surface Antigen on the Risk of Liver Metastasis: A Retrospective Clinical Study of 4033 Consecutive Cases of Newly Diagnosed Colorectal Cancer. Clin Infect Dis. 2018; 66: 1948-52.

9. Terrault NA, Lok ASF, McMahon BJ, Chang KM, Hwang JP, Jonas MM, et al. Update on Prevention, Diagnosis, and Treatment of Chronic Hepatitis B: AASLD 2018 Hepatitis B Guidance. Clin Liver Dis (Hoboken). 2018; 12: 33-4.

10. Lian JQ, Yang XF, Zhao RR, Zhao YY, Li Y, Zhang Y, et al. Expression profiles of circulating cytokines, chemokines and immune cells in patients with hepatitis B virus infection. Hepat Mon. 2014; 14: e18892.

11. Halama N, Zoernig I, Berthel A, Kahlert C, Klupp F, Suarez-Carmona M, et al. Tumoral Immune Cell Exploitation in Colorectal Cancer Metastases Can Be Targeted Effectively by Anti-CCR5 Therapy in Cancer Patients. Cancer Cell. 2016; 29: 587-601.

12. Toiyama Y, Fujikawa H, Kawamura M, Matsushita K, Saigusa S, Tanaka K, et al. Evaluation of CXCL10 as a novel serum marker for predicting liver metastasis and prognosis in colorectal cancer. Int J Oncol. 2012; 40: 560-6.

13. Iwata $T$, Tanaka $K$, Inoue $Y$, Toiyama $Y$, Hiro J, Fujikawa $H$, et al. Macrophage inflammatory protein-3 alpha (MIP-3a) is a novel serum prognostic marker in patients with colorectal cancer. J Surg Oncol. 2013; 107: 160-6.

14. Ghadjar P, Coupland SE, Na IK, Noutsias M, Letsch A, Stroux A, et al. Chemokine receptor CCR6 expression level and liver metastases in colorectal cancer. J Clin Oncol. 2006; 24: 1910-6.

15. Chen HD, Li N, Ren JS, Shi JF, Zhang YM, Zou SM, et al. [Compliance rate of screening colonoscopy and its associated factors among high-risk populations of colorectal cancer in urban China]. Zhonghua Yu Fang Yi Xue Za Zhi. 2018; 52: 231-7.

16. Levin TR, Corley DA, Jensen CD, Schottinger JE, Quinn VP, Zauber AG, et al. Effects of Organized Colorectal Cancer Screening on Cancer Incidence and Mortality in a Large Community-Based Population. Gastroenterology. 2018; 155: 1383-91 e5.

17. Manfredi S, Lepage C, Hatem C, Coatmeur O, Faivre J, Bouvier AM. Epidemiology and management of liver metastases from colorectal cancer. Ann Surg. 2006; 244: 254-9. 